\title{
A manufacturing process for an energy storage device using $3 D$ printing
}

\author{
Anan Tanwilaisiri*, Ruirong Zhang, Yanmeng Xu, David Harrison and John Fyson \\ College of Engineering, Design and Physical Sciences Brunel University London, UK
}

anan.tanwilaisiri@brunel.ac.uk

\begin{abstract}
D printing has been widely applied in the development of prototypes. The main advantage of this process is that the objects or products can be viewed in three dimensions on a computer display and a 3D sample can be created before committing to a large production run. There are various 3D printing technologies that are capable of manufacturing metal, ceramic, plastic substrate and paste objects. Recently several research groups have focused on the fabrication freedom of $3 D$ printing for different purposes including freeform manufacturing of electrochemical devices but this use is still limited. This paper describes a manufacturing process for electrochemical supercapacitors using the combination of the two techniques of 3D printing which are Fused Deposition Modelling (FDM) and a Paste Extrusion system. The method relies on creating a frame for the energy storage device, i.e. supercapacitor, by the FDM 3D printer and then depositing the conductive layers and electrodes of the supercapacitor using Paste Extrusion system. A 3D supercapacitor has been made and evaluated in this study.
\end{abstract}

Keywords-3D printing, supercapacitors, fused deposition modeling printing, paste extrusion system

\section{INTRODUCTION}

Energy has become a main focus of the world powers and the scientific community. More efficient energy storage devices have attracted great attention. One such device, the supercapacitor, has increased in use significantly over the last decade and has the potential to facilitate significant advances in energy storage [1-3]. Supercapacitors, also called ultracapacitors or electrochemical capacitors, have several advantages for energy storage devices such as high power densities, long life cycles and high reversibility. Because of these capabilities, they have been applied to portable electronic devices, electric vehicles and emergency power supplies. There are two common types of supercapacitors: electrochemical double layer capacitors (EDLCs) and pseudocapacitors. For EDLCs, the energy is stored by the formation of separated electric charges at the interface of the porous electrode material and the electrolyte. For pseudocapacitors, electrons are transferred between the electrolyte and the electrode through fast Faradiac redox reactions in order to generate current. In order to print energy storage devices, such as supercapacitors, there has been recently considerable interest in adapting the additive manufacturing process that is usually applied in the rapid prototyping industry [4-6].

Additive manufacturing (AM) is a term to explain technology applied to the rapid production of 3D objects which is becoming increasingly capable and affordable. The process of $3 \mathrm{D}$ printing or $\mathrm{AM}$ is used to fabricate $3 \mathrm{D}$ objects or products on a layer by layer basis. The advantage of the 3D printing technique is its ability to create different structures for many applications in several different materials rapidly. At present, the technologies of additive manufacturing allow for the creation of printed parts and not just models. Many researchers recently have aimed to develop new techniques and improve novel materials in order to create a wide range of applications for different purposes [7-9].

Nowadays, the developed component placement and electrical interconnect deposition applied in advanced 3D printing technology can offer electronic prototypes. Current research in $3 \mathrm{D}$ printing processes aims to integrate electronic components and interconnects into 3D printed devices. This 3D electronics integration is also known as 3D structural electronics or 3D printed electronics. 3D printed electronics can adapt to any figure offering a unique improvement over conventional electronics system. Studies in embedding electronic components and electrical interconnections into 3D structures are being reported. The 3D printed structure embedded with conductive ink using Direct Printing (DP) was presented by Palmer and expanded in Medina and Lopes [1015]. In their research, a stereolithography (SL) machine was integrated with a dispensing system and applied to print simple circuits, including a demonstration of simple prototype temperature sensors. For 3D printed energy storage devices, 3D interdigitated microbattery architectures (3D-IMA) were introduced by Sun et al. [16]. The 3D-IMA was comprised of $\mathrm{Li}_{4} \mathrm{Ti}_{5} \mathrm{O}_{12}$ (LTO) and $\mathrm{LiFePO}_{4}$ (LFP) applied as anode and cathode material respectively. In addition, Zhao et al [17] presented a 3D printed electrode for fabricating interdigitated supercapacitors using Selective Laser Melting (SLM).

In this work, we report the combination of FDM and a Paste Extrusion system for manufacturing a supercapacitor. FDM technology was used to print a 3D structure of supercapactiors and a Paste Extrusion system was used to print a current collector layer and an electrode. The method demonstrated in this paper opens up a novel approach for manufacturing embedded energy storage devices.

\section{EXPERIMENT}

\section{A. Printer and Material used}

The 3D printer used in this work was a FDM printing machine, an Ultimaker2. The advantage of this FDM technology is it provides a simple fabrication process, reliability, low cost of material and the accessibility to a wide 
range of building thermoplastic. This FDM machine is attached to an extra extruder which is a paste extrusion system. This extra extruder used a syringe to deposit different paste materials for this experiment. The material deposit by the main extrusion head of FDM machine was polylactic acid (PLA).

The ideal conductive current collector material is low cost, nontoxic, and commonly available, and its viscosity and curing conditions should be compatible with the deposition system used. Silver conductive paint purchased from RS Components Ltd was used as the current collector material in this study. The electrode material of activated carbon was made into slurry mixed by the carbon material with carboxy methyl cellulose (CMC) as a binder. CMC binder was prepared by mixing a certain amount of $\mathrm{CMC}$ with water/ethanol (1:1) solvent at room temperature. It was stirred overnight on a magnetic stirrer. The concentration of CMC was fixed at $10 \mathrm{wt} \%$ based on total mass of $\mathrm{AC}$ and $\mathrm{CMC}$. Then $0.5 \mathrm{~g}$ activated carbon was added to the CMC binder solution. In order to obtain an uniform slurry, it was stirred for 8 hours.

The electrolyte applied in this experiment was a mixture of tetrabutylammonium tetrafluoroborate $\left(\mathrm{C}_{16} \mathrm{H}_{36} \mathrm{BF}_{4} \mathrm{~N}\right)$ with propylene carbonate $\left(\mathrm{C}_{4} \mathrm{H}_{6} \mathrm{O}_{3}\right)$. The concentration of the electrolyte used was fixed at $1 \mathrm{~mol} / \mathrm{L}$.

\section{B. Manufacture of supercapacitor using the $3 D$ printer}

\section{1) Design and printed with FDM printing}

The supercapacitors designed for this study comprise of two electrodes and a separator layer with soaked in electrolyte. Figure 1 shows the schematic of the crosssection of the supercapacitor structure. The 3D frame for the supercapacitor was designed using Solidworks software and created by the $3 \mathrm{D}$ printing machine. A view of the frame is shown in Figure 2.

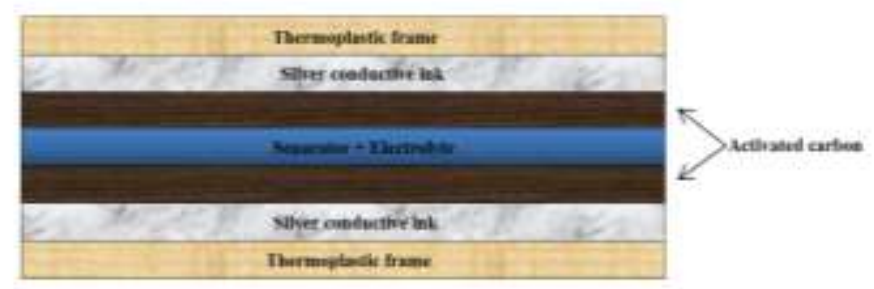

Fig. 1 Schematic of design structure of supercapacitor.

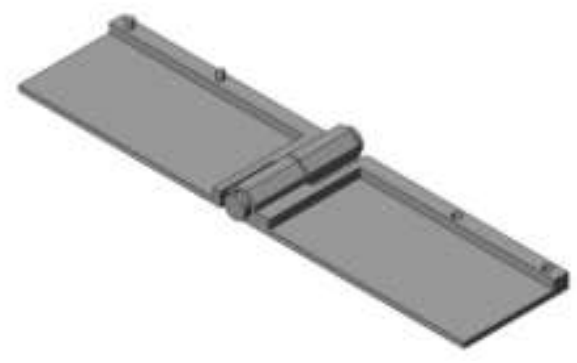

Fig. 2 The 3D frame for the supercapacitor

The 3D frame for the supercapacitor was designed and similar to a hinge with a size of $3 \times 5 \mathrm{~cm}$. It can be printed in one printing event and requires no assembly. In addition, this design makes it easy to form the supercapacitor when all layers are finally printed.

The software used to set up all specification of printing including transforming STL file to g-code was CURA 15.04. For printing this object, the height of each layer was adjusted to $0.1 \mathrm{~mm}$. The printing speed was $50 \mathrm{~mm} / \mathrm{sec}$. The material in this printing was PLA rod with diameter of $2.89 \mathrm{~mm}$. The 3D frame structure of the supercapacitor was fabricated by the main extrusion head of the printer. The PLA material was heated and became semi-liquid. Then it was deposited layer by layer in ultra-fine beads along the extrusion path. The temperature of extruder was controlled at $220^{\circ} \mathrm{C}$ and $60{ }^{\circ} \mathrm{C}$ at the bed of the printer.

\section{2) Printing current collector and electrode}

The paste extrusion system was used to print two materials. The 3D packaging frame was printed first with silver conductive paint. The conductive paint was printed three times with a size of $3 \times 5.5 \mathrm{~cm}$ on each side of the 3D frame objects in order to build the conductive current collector layer. Then the sample was dried at room temperature after each of these layers was printed to enhance the adhesion of the carbon slurry layer to be coated onto it.

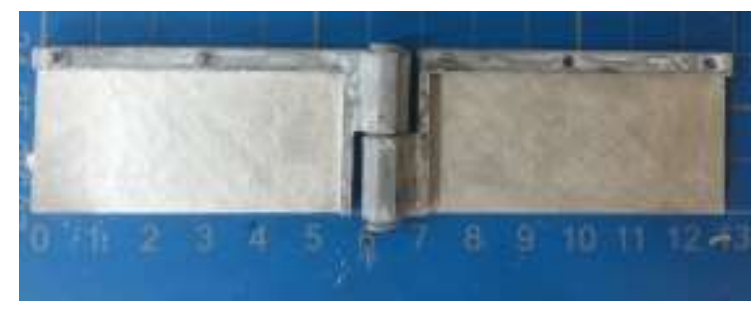

Fig. 3 Paste Extrusion system prints the silver conductive ink on $3 \mathrm{D}$ packaging frame structure.

The slurry was then printed on the top of the area of the silver conductive paint with a size of $2.5 \times 4 \mathrm{~cm}$. It was printed five times in the same position and dried at room temperature as shown in Figure 4 (a). These slurry layers were applied as the material for the electrodes. The electrolyte was dropped on the surface of the printed activated materials, and then these 
electrodes with absorbed electrolyte were held under vacuum for 0.5 hour in order to cause the electrolyte to be fully in contact with the carbon material. In the meantime, a filter paper was wetted with the electrolyte and used as the separator. The two coated electrodes were folded over to sandwich the filter paper separator, and an assembled electrochemical supercapacitor was achieved as shown in Figure 4 (b).

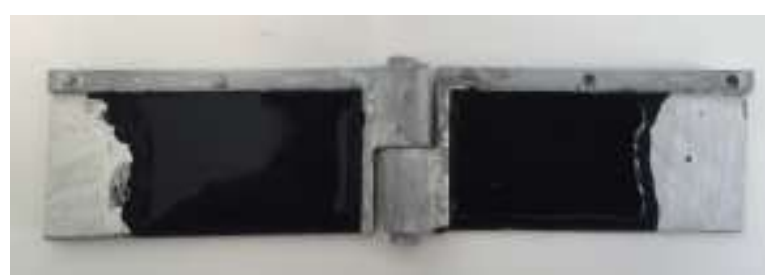

(a)

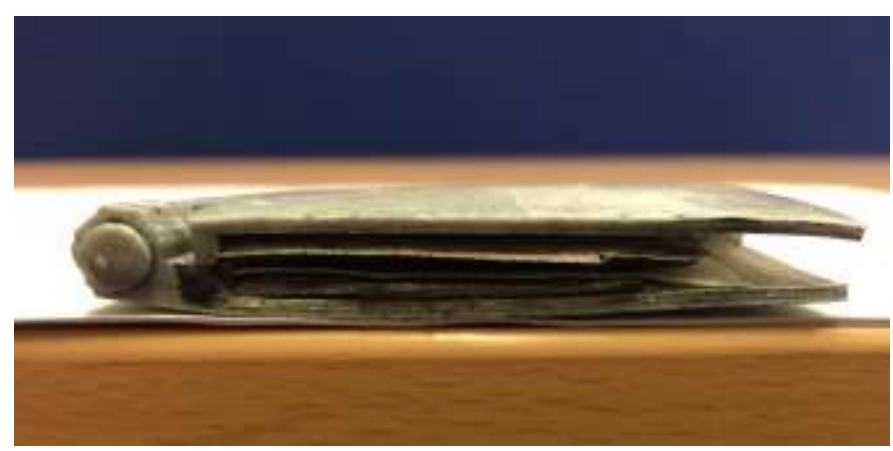

(b)

Fig. 4 Printed AC material as electrodes for the final electrochemical supercapacitor.

(a) Showing the slurry printed on 3D object after printed the silver conductive ink.

(b) Side view of 3D supercapacitor.

\section{RESULTS AND DICUSSION}

When applying the current to EDLC, opposite charges accumulate on the surfaces of the electrodes. The separator in EDLC is used to avoid short-circuits caused by contact of the two electrodes. The function of energy storage is accomplished after an electric potential is applied between the two electrodes. A cyclic voltammogram (CV) test was performed by using a positive (charging) voltage sweep $\mathrm{d} V / \mathrm{d} t$ (scan rate) in a definite current range and reversing (discharging) the current sweep opposition immediately after the maximum voltage is complete.

The CV of the prototype supercapacitor was characterized using an electrochemical workstation-VersaStat 3. As shown in Figure 5, the electrochemical character of a 3D printed supercapacitor is measured according to the corresponding current response against the applied voltage. The curve shape shows that the device operated as a supercapacitor. From the Figure, the capacitance, $C 1$, of the $3 \mathrm{D}$ printed EDLC can be calculated by the following equation (1):

$$
C 1=\frac{\text { Qtotal }}{2 \Delta V}
$$

Where $Q_{\text {total }}$ is the supercapacitor's charge in coulombs. This value is measured by the $\mathrm{CV}$ system used. $\Delta V$ is the voltage change between the device's terminals in volts (V).

The capacitance calculated was $0.212 \mathrm{~F}$. The specific capacitance of the supercapacitor was about $0.20 \mathrm{~F} / \mathrm{g}$. These results demonstrate that the manufacturing process for the supercapacitor printed by $3 \mathrm{D}$ printing illustrated above is a satisfactory fabrication method.

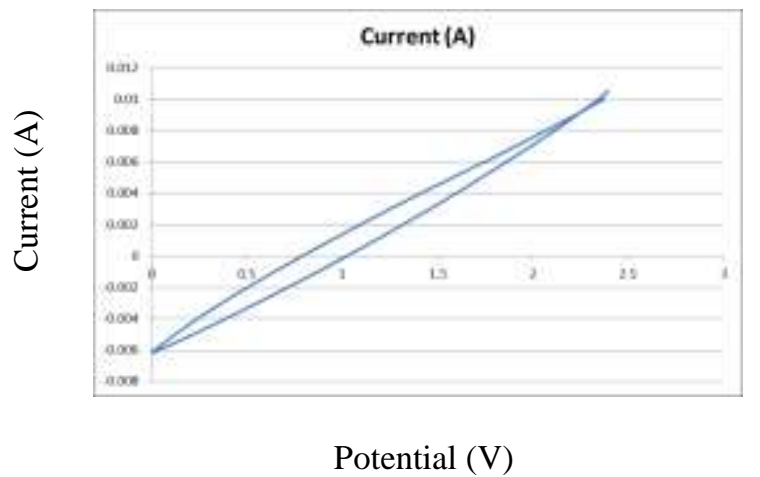

Fig. 5 The cyclic voltammogram recorded of the 3D printed supercapacitors.

\section{CONCLUSION AND FUTURE WORK}

The study has demonstrated the first trial of a manufacturing method of combining the two 3D printing technologies, i.e. a FDM system to create the frame and a paste extrusion system to print conductive current collectors and electrodes, to fabricate an electrochemical double layer capacitor. However, the electrical performance and packaging frame of the supercapacitor may be improved by optimizing the manufacturing processes with different materials in the future. For example, it might be able to increase the electrical performance when each printed layer is consistently and uniformly controlled.

\section{REFERENCES}

[1] Vangari, M., Pryor, T. and Jiang, L. (2012) "Supercapacitors: Review of materials and fabrication methods", Journal of Energy Engineering, vol. 139 , no. 2 , pp. $72-79$.

[2] Calvert, P. (2001) "Inkjet printing for materials and devices", Chemistry of Materials, vol. 13, no. 10, pp. 3299-3305. 
[3] Perelaer, J., Smith, P.J., Mager, D., Soltman, D., Volkman, S.K., Subramanian, V., Korvink, J.G. and Schubert, U.S. (2010) "Printed electronics: the challenges involved in printing devices, interconnects, and contacts based on inorganic materials", Journal of Materials Chemistry, vol. 20, no. 39, pp. 8446-8453.

[4] BOICEA, V.A., 2014. Energy Storage Technologies: The Past and the Present. Proceedings of the IEEE, 102(11), pp. 1777-1794.

[5] KIEBELE, A., KAEMPGEN, M. and GRUNER, G., 2008. Printed Energy and Power Storage: Batteries and Supercapacitors. Nanotech.L.\& Bus., 5, pp. 7.

[6] Conédéra, V., Mesnilgrente, F., Brunet, M. and Fabre, N. (2009) "Fabrication of activated carbon electrodes by inkjet deposition", Quantum, Nano and Micro Technologies, 2009. ICQNM'09. Third International Conference onIEEE, , pp. 157.

[7] HUANG, S.H., LIU, P., MOKASDAR, A. and HOU, L., 2013. Additive manufacturing and its societal impact: a literature review. The International Journal of Advanced Manufacturing Technology, 67(5-8), pp. 1191-1203.

[8] KRUTH, J., LEU, M. and NAKAGAWA, T., 1998. Progress in additive manufacturing and rapid prototyping. CIRP Annals-Manufacturing Technology, 47(2), pp. 525-540.

[9] VAEZI, M., SEITZ, H. and YANG, S., 2013. A review on 3D microadditive manufacturing technologies. The International Journal of Advanced Manufacturing Technology, 67(5-8), pp. 1721-1754.

[10] Czyżewski, J., Burzyński, P., Gaweł, K. and Meisner, J. (2009) 'Rapid prototyping of electrically conductive components using 3D printing technology', Journal of Materials Processing Technology, 209(12-13), pp. 5281-5285.

[11]Espalin, D., Muse, D.W., MacDonald, E. and Wicker, R.B. (2014) '3D Printing multifunctionality: structures with electronics', The International Journal of Advanced Manufacturing Technology, 72(5-8), pp. 963-978

[12]Leigh, S.J., Bradley, R.J., Purssell, C.P., Billson, D.R. and Hutchins, D.A (2012) 'A simple, low-cost conductive composite material for 3D printing of electronic sensors', PloS one, 7(11), pp. e49365.

[13] Ready, S., Endicott, F., Whiting, G.L., Ng, T.N., Chow, E.M. and Lu, J. (2013) '3D Printed Electronics', NIP \& Digital Fabrication Conference. Society for Imaging Science and Technology, 9-12.

[14] Aguilera, E., Ramos, J., Espalin, D., Cedillos, F., MuseP , D., Wicker, R. and MacDonald, E. '3D Printing of Electro Mechanical Systems', .

[15] SARIK, J., BUTLER, A., SCOTT, J., HODGES, S. and VILLAR, N., Combining 3D printing and printable electronics.

[16] Sun, K., Wei, T., Ahn, B.Y., Seo, J.Y., Dillon, S.J. and Lewis, J.A. (2013) '3D Printing of Interdigitated Li-Ion Microbattery Architectures', Advanced Materials, 25(33), pp. 4539-4543.

[17]ZHAO, C., WANG, C., GORKIN III, R., BEIRNE, S., SHU, K. and WALLACE, G.G., 2014. Three dimensional (3D) printed electrodes for interdigitated supercapacitors. Electrochemistry Communications, 41, pp. 20-23 